\title{
An Observer-Based PID Regulator
}

\author{
L. Keviczky, Cs. Bányász \\ Széchenyi István University, Győr \\ Computer and Automation Research Institute and \\ MTA-BME Control Engineering Research Group \\ Hungarian Academy of Sciences \\ H-1111 Budapest, Kende u 13-17, HUNGARY \\ Phone: +361-466-5435; Fax: +361-466-7503 \\ e-mail: keviczky@sztaki.hu; banyasz@sztaki.hu
}

\begin{abstract}
An equivalent transfer function representation (TFR) is used to study the state-feedback/observer $(S F O)$ topologies of control systems. This approach is applied to combine this methodology with YOULAparametrization $(Y P)$ introducing new classes of regulators. Then this method is used to introduce observer based PID regulators.
\end{abstract}

Keywords: Observer, state-feedback, model error, YoULA-parametrization, PID regulator

\section{Introduction}

In our previous paper [6] it was shown that in the classical state-feedback/observer $(S F O)$ scheme the model error decreases by the sensitivity function of the observer feedback loop. An equivalent transfer function representation (TFR) was used to demonstrate this special feature of these regulators. It was also shown that this principle can be used to generalize for the YoulA-parametrized regulators, too. In this paper the demonstrated new approach is used to introduce further new class of regulators.

\section{The Observer Based Youla-Regulator}

For open-loop stable processes the all realizable stabilizing $(A R S)$ model based regulator $\hat{C}$ is the YoULA-parametrized one:

$$
\hat{C}(\hat{P})=\left.\frac{Q}{1-Q \hat{P}}\right|_{\hat{P} \rightarrow P}=\frac{Q}{1-Q P}=C(P),
$$

where the "parameter" $Q$ ranges over all proper $(Q(\omega=\infty)$ is finite), stable transfer functions [5], [7], see Fig. 1a.

It is important to know that the $Y$-parametrized closed-loop with the $A R S$ regulator is equivalent to the well-known form of the so-called Internal Model Control (IMC) 
principle [7] based structure shown in Fig. 1b.

$Q$ is anyway the transfer function from $r$ to $u$ and the closed-loop transfer function (i.e., $C S F$ ) for $\hat{P}=P$, when $\ell \rightarrow 0$

$$
\hat{T}_{\text {ry }}=\frac{\hat{C} P}{1+\hat{C} P}=\left.Q P \frac{1+\ell}{1+(1-Q P) \ell}\right|_{\ell \rightarrow 0}=Q P=T_{\text {ry }}
$$

is linear (and hence convex) in $Q$.

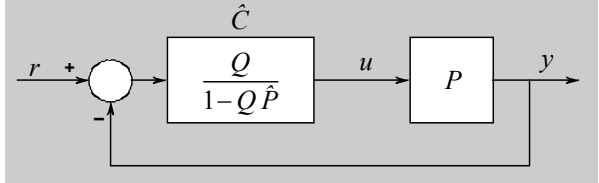

(a)

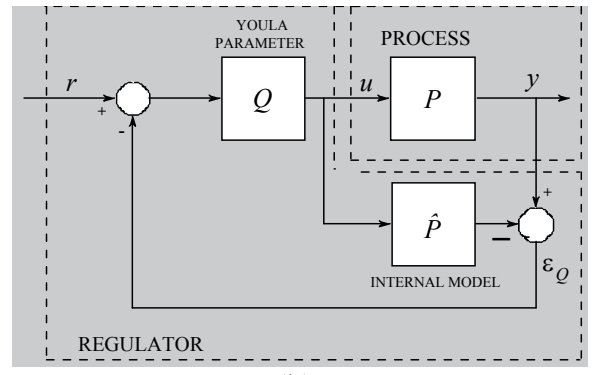

(b)

Figure 1. The equivalent IMC structure of an ARS regulator

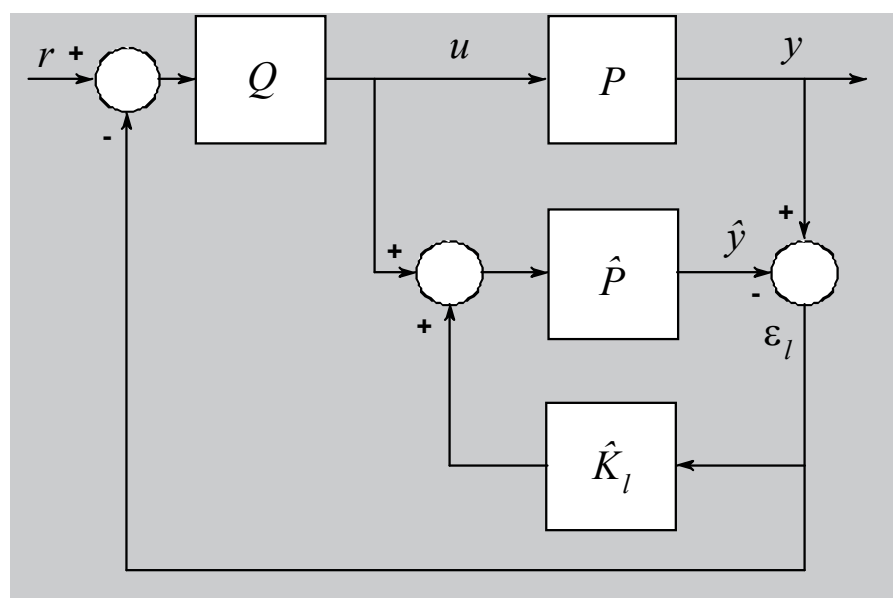

Figure 2. The observer-based IMC structure

It is interesting to compute the relative error $\ell_{\mathrm{T}}$ of $\hat{T}_{\mathrm{ry}}$

$$
\ell_{\mathrm{T}}=\frac{T_{\mathrm{ry}}-\hat{T}_{\mathrm{ry}}}{\hat{T}_{\mathrm{ry}}}=\frac{T_{\mathrm{ry}}}{\hat{T}_{\mathrm{ry}}}-1=\frac{Q P}{\frac{Q P}{1-Q(P-\hat{P})}}-1=Q(P-\hat{P})=Q P \frac{\ell}{1+\ell}=T_{\mathrm{ry}} \frac{\ell}{1+\ell} .
$$


The equivalent $I M C$ structure performs the feedback from the model error $\varepsilon_{Q}$. Similarly to the $S F O$ scheme it is possible to construct an internal closed-loop, which virtually reduces the model error to

$$
\varepsilon_{l}=\frac{1}{1+\hat{K}_{l} \hat{P}}(y-\hat{P} u)=\frac{1}{1+\hat{K}_{l} \hat{P}} \varepsilon_{Q}=\frac{1}{1+\hat{L}_{l}} \varepsilon_{Q}=\hat{H} \varepsilon_{Q} ; \quad \hat{L}_{l}=\hat{K}_{l} \hat{P}
$$

and performs the feedback from $\varepsilon_{l}$ (see Fig. 2), where $\hat{L}_{l}$ is the internal loop transfer function. In this case the resulting closed-loop will change to the scheme shown in Fig. 3.

This means that the introduction of the observer feedback changes the YOULAparametrized regulator to

$$
\hat{C}^{\prime}\left(\hat{P}^{\prime}\right)=\frac{Q}{1-Q \frac{\hat{P}}{1+\hat{K}_{l} \hat{P}}}=\frac{Q\left(1+\hat{K}_{l} \hat{P}\right)}{1+\hat{K}_{l} \hat{P}-Q \hat{P}} .
$$

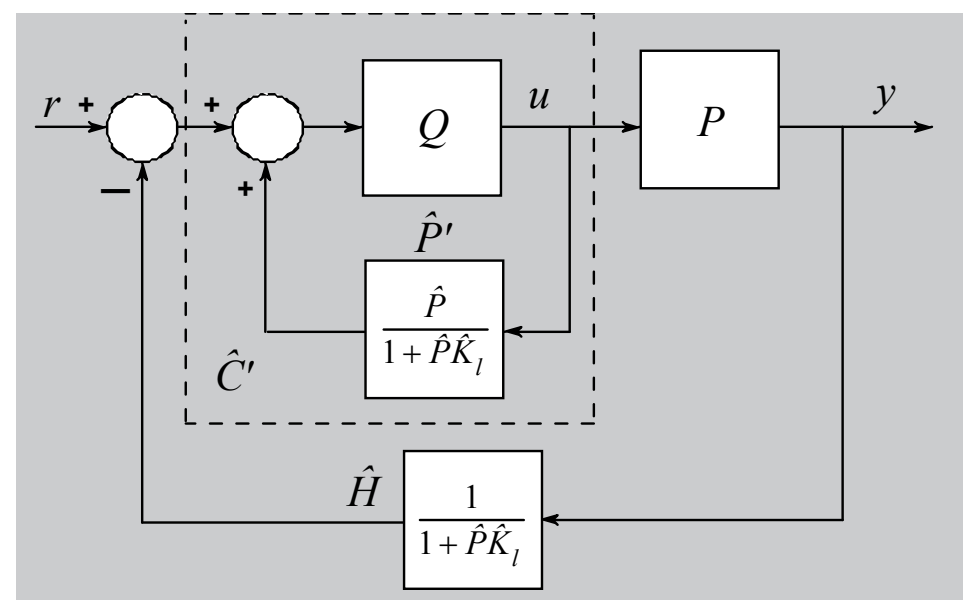

Figure 3. Equivalent closed-loop for the observer-based IMC structure

The form of $\hat{C}^{\prime}$ shows that the regulator virtually controls a fictitious plant $\hat{P}^{\prime}$, which is also demonstrated in Fig. 3. Here the fictitious plant is

$$
\hat{P}^{\prime}=\frac{\hat{P}}{1+\hat{K}_{l} \hat{P}}=\frac{\hat{P}}{1+\hat{L}_{l}} .
$$


The closed-loop transfer function is now

$$
T_{\mathrm{ry}}^{\prime}=\frac{\hat{C}^{\prime} P}{1+\hat{C}^{\prime} P}=\frac{Q P\left(1+\hat{K}_{l} \hat{P}\right)}{1+\hat{K}_{l} \hat{P}-Q \hat{P}+Q P}=\left.Q P \frac{1}{1+Q P \frac{1}{1+\hat{K}_{l} \hat{P}} \frac{\ell}{1+\ell}}\right|_{\ell \rightarrow 0}=Q P=T_{\mathrm{ry}} .
$$

The relative error $\ell_{\mathrm{T}}^{\prime}$ of $\hat{T}_{\mathrm{ry}}^{\prime}$ becomes

$$
\ell_{\mathrm{T}}^{\prime}=\frac{T_{\mathrm{ry}}-\hat{T}_{\mathrm{ry}}^{\prime}}{\hat{T}_{\mathrm{ry}}^{\prime}}=\frac{T_{\mathrm{ry}}}{\hat{T}_{\mathrm{ry}}^{\prime}}-1=\frac{Q P}{\frac{Q P\left(1+\hat{K}_{l} \hat{P}\right)}{1+Q(P-\hat{P})+\hat{K}_{l} \hat{P}}}-1=Q P \frac{\ell}{1+\ell} \frac{1}{1+\hat{K}_{l} \hat{P}}=\ell_{\mathrm{T}} \frac{1}{1+\hat{L}_{l}}
$$

which is smaller than $\ell_{\mathrm{T}}$. The reduction is by $\hat{H}=1 /\left(1+\hat{L}_{l}\right)$.

\section{An Observer Based PID-Regulator}

The ideal form of a YOULA-regulator based on reference model design [5] is

$$
C_{\mathrm{id}}=\frac{\left(R_{\mathrm{n}} P^{-1}\right)}{1-\left(R_{\mathrm{n}} P^{-1}\right) P}=\frac{Q}{1-Q P}=\frac{R_{\mathrm{n}}}{1-R_{\mathrm{n}}} P^{-1},
$$

when the inverse of the process is realizable and stable. Here the operation of $R_{\mathrm{n}}$ can be considered a reference model (desired system dynamics). It is generally required that the reference model has to be strictly proper with unit static gain, i.e., $R_{\mathrm{n}}(\omega=0)=1$.

For a simple, but robust PID regulator design method assume that the process can be well approximated by its two major time constants, i.e.,

$$
P \cong \frac{A}{\mathrm{~A}_{2}}
$$

where

$$
\mathrm{A}_{2}=\left(1+s T_{1}\right)\left(1+s T_{2}\right)
$$

According to (9) the ideal YoULA-regulator is 


$$
C_{\mathrm{id}}=\frac{R_{\mathrm{n}} P^{-1}}{1-R_{\mathrm{n}}}=\frac{R_{\mathrm{n}}\left(1+s T_{1}\right)\left(1+s T_{2}\right)}{A\left(1-R_{\mathrm{n}}\right)} ; \quad T_{1}>T_{2} .
$$

Let the reference model $R_{\mathrm{n}}$ be of first order

$$
R_{\mathrm{n}}=\frac{1}{1+s T_{\mathrm{n}}},
$$

which means that the first term of the regulator is an integrator

$$
\frac{R_{\mathrm{n}}}{1-R_{\mathrm{n}}}=\frac{\frac{1}{1+s T_{\mathrm{n}}}}{1-\frac{1}{1+s T_{\mathrm{n}}}}=\frac{1}{1+s T_{\mathrm{n}}-1}=\frac{1}{s T_{\mathrm{n}}},
$$

whose integrating time is equal to the time constant of the reference model. Thus the resulting regulator corresponds to the design principle, i.e., it is an ideal PID regulator

$$
C_{\mathrm{PID}}=A_{\mathrm{PID}} \frac{\left(1+s T_{\mathrm{I}}\right)\left(1+s T_{\mathrm{D}}\right)}{s T_{\mathrm{I}}}=A_{\mathrm{PID}} \frac{\left(1+s T_{1}\right)\left(1+s T_{2}\right)}{s T_{1}}
$$

with

$$
A_{\mathrm{PID}}=\frac{T_{1}}{A T_{\mathrm{n}}} ; \quad T_{\mathrm{I}}=T_{1} ; \quad T_{\mathrm{D}}=T_{2} .
$$

The YoulA-parameter $Q$ in the ideal regulator is

$$
Q=R_{\mathrm{n}} P^{-1}=\frac{1}{A} \frac{\left(1+s T_{1}\right)\left(1+s T_{2}\right)}{1+s T_{\mathrm{n}}} .
$$

It is not necessary, but desirable to ensure the realizability, i.e., it is reasonable to use

$$
Q=R_{\mathrm{n}} P^{-1}=\frac{1}{A} \frac{\left(1+s T_{1}\right)\left(1+s T_{2}\right)}{\left(1+s T_{\mathrm{n}}\right)(1+s T)},
$$

where $T$ can be considered as the time constant of the derivative action $\left(0.1 T_{\mathrm{D}} \leq T \leq 0.5 T_{\mathrm{D}}\right)$. The regulator $\hat{C}^{\prime}$ and the feedback term $\hat{H}$ must be always realizable. In the practice the PID regulator and the YouLA-parameter is always modelbased, so

$$
\begin{gathered}
\hat{C}_{\mathrm{PID}}(\hat{P})=\hat{A}_{\mathrm{PID}} \frac{\left(1+s \hat{T}_{1}\right)\left(1+s \hat{T}_{2}\right)}{s \hat{T}_{1}} ; \quad \hat{A}_{\mathrm{PID}}=\frac{\hat{T}_{1}}{\hat{A} T_{\mathrm{n}}}, \\
\hat{Q}=R_{\mathrm{n}} \hat{P}^{-1}=\frac{1}{\hat{A}} \frac{\left(1+s \hat{T}_{1}\right)\left(1+s \hat{T}_{2}\right)}{1+s T_{\mathrm{n}}} .
\end{gathered}
$$




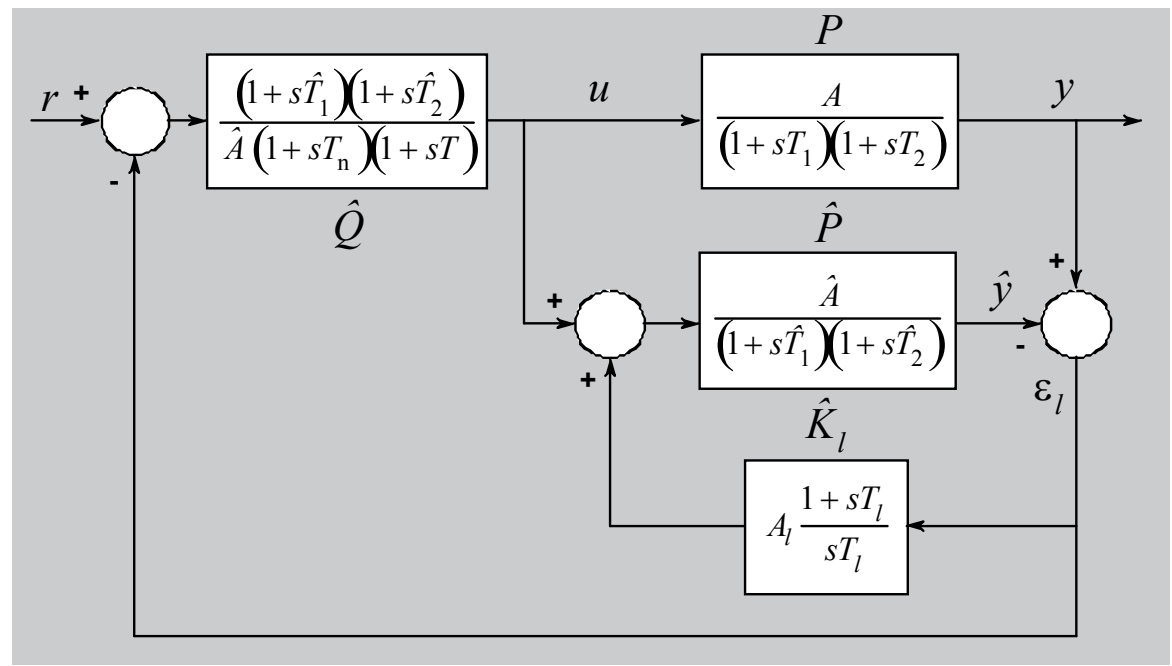

Figure 4. An observer based PID regulator

The scheme of the observer based PID regulator is shown in Fig. 4, where a simple PI regulator

$$
\hat{K}_{l}=A_{l} \frac{1+s T_{l}}{s T_{l}}
$$

is applied in the observer-loop. Here $T_{l}$ must be in the range of $T$, i.e., considerably smaller than $T_{1}$ and $T_{2}$.

Note that the frequency characteristic of $\hat{H}$ cannot be easily designed to reach a proper error suppression. For example, it is almost impossible to design a good realizable high cut filter in this architecture. The high frequency domain is always more interesting to speed up a control loop, so the target of the future research is how to select $\hat{K}_{l}$ for the desired shape of $\hat{H}$.

\section{Simulation Experiments}

The simulation experiments were performed in using the observer based PID scheme shown in Fig. 4. 


\section{Example 1}

The process parameters are: $T_{1}=20, T_{2}=10$ and $A=1$. The model parameters are: $\hat{T}_{1}=25, \hat{T}_{2}=12$ and $\hat{A}=1.2$. The purpose of the regulation is to speed up the basic step response by 4 , i.e., $T_{n}=5$ is selected in the first order $R_{n}$. In the observer loop a simple proportional regulator $\hat{K}_{l}=0.01$ is applied. The ideal form of $Q(17)$ was used. Figure 5 shows some step responses in the operation of the observer based PID regulator.

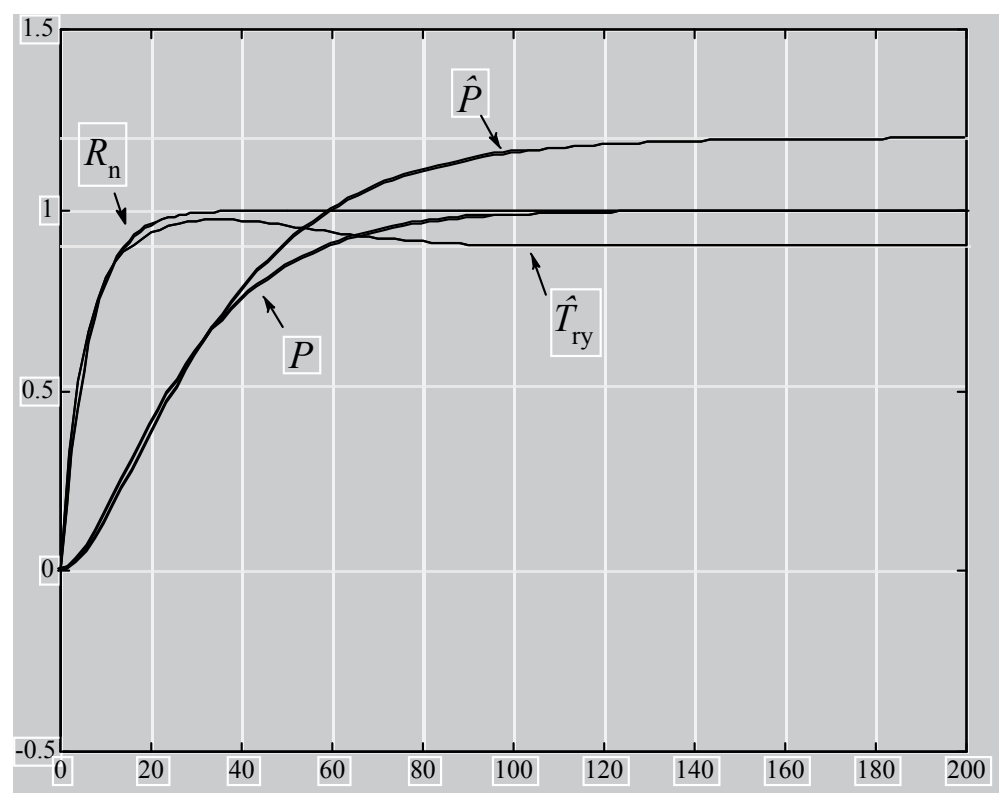

Figure 5. Step responses using the observer based PID regulator

It is easy to see that the $\hat{T}_{\text {ry }}^{\prime}$ very well approximates $R_{n}$ in the high frequencies (for small time values) in spite of the very bad model $\hat{P}$. 


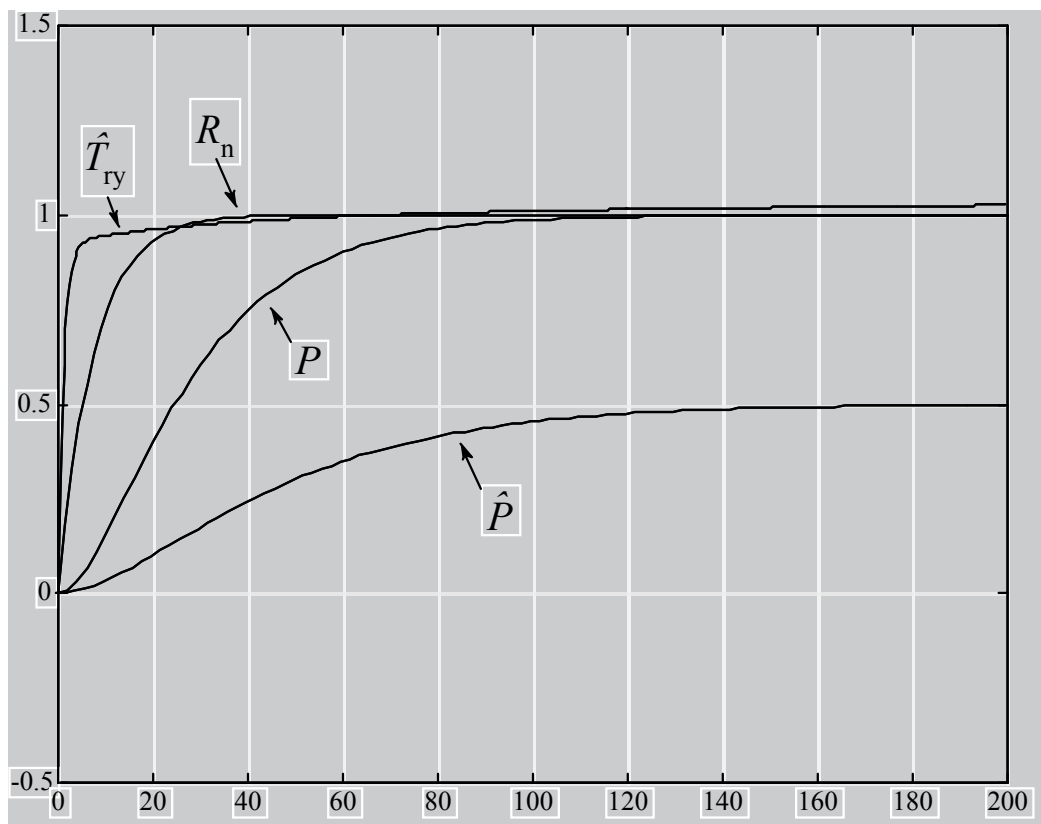

Figure 6. Step responses using the observer based PID regulator

\section{Example 2}

The process parameters and the selected first order $R_{n}$ are the same as in the previous example. The model parameters are: $\hat{T}_{1}=30, \hat{T}_{2}=20$ and $\hat{A}=0.5$. In the observer loop a $P I$ regulator (67) is applied with $A_{l}=0.001$ and $T_{l}=2$. The ideal form of $Q$ (17) was used. Figure 6 shows some step responses in the operation of the observer based $P I D$ regulator.

It is easy to see that the $\hat{T}_{\mathrm{ry}}^{\prime}$ well approximates $R_{n}$ in the high frequencies (for small time values) in spite of the very bad model $\hat{P}$.

\section{Conclusions}

It was shown that the $S F O$ methodology can be applied to the YouLA-parametrized regulators, too. This approach reduces the model error by the sensitivity function of the observer loop similarly to the $S F O$ scheme.

Using this new approach an observer based PID regulator was also introduced. This regulator works well even in case of large model errors as some simulations showed. 


\section{Aknowledgement}

This work was supported in part by the MTA-BME Control Engineering Research Group of the HAS, at the Budapest University of Technology and Economics and by the project TAMOP 4.2.2.A-11/1/KONV-2012-2012, at the Széchenyi István University of Györ.

\section{References}

[1] Åström K.J., B. Wittenmark: Computer Controlled Systems, Prentice-Hall, 1984

[2] Åström K.J.: Control System Design Lecture Notes, University of California, Santa Barbara, 2002

[3] Kailath T.: Linear Systems, Prentice Hall, 1980

[4] Keviczky L.: Combined identification and control: another way, Control Engineering Practice, vol. 4, no. 5, pp. 685-698, 1996

DOI: 10.1016/0967-0661(96)00052-4

[5] Keviczky L., Cs. Bányász: Iterative identification and control design using $K-B$ parametrization, In: Control of Complex Systems, Eds: K.J. Åström, P. Albertos, M. Blanke, A. Isidori, W. Schaufelberger, R. Sanz, Springer, pp. 101-121, 2001

[6] Keviczky L., Cs. Bányász: Attenuation of the Model Error in Observer-Based State-Feedback Regulators, Acta Technica Jaurinensis, vol. 7, vo. 1, pp. 46-61, 2014

DOI: 10.14513/actatechjaur.v7.n1.256

[7] Maciejowski J.M.: Multivariable Feedback Design, Addison Wesley, 1989 